\title{
Estilos de aprendizaje en estudiantes universitarios: una revisión descriptiva de la literatura
}

\author{
Learning styles in university students: A descriptive review of literature
}

\author{
Cira Thania Porras-Mucha ${ }^{1, a}$ \\ https://orcid.org/0000-0001-6751-5648 \\ Isabel Angela Carhuas-Arzapalo 1,a \\ https://orcid.org/0000-0003-3962-7073
}

\author{
Maira Quispe-Paiva ${ }^{1, a}$ \\ https://orcid.org/0000-0003-4189-6352 \\ Claudia Ríos-Cataño ${ }^{1,6}$ \\ https://orcid.org/0000-0001-7547-9093
}

\section{Citar como}

Porras Mucha, C., Carhuas, I., Quispe, M. y Ríos, C. (2021, mayo 18). Estilos de aprendizaje en estudiantes universitarios: una revisión descriptiva de la literatura. Desafíos, 12(2); 109-16. https://doi.org/10.37711/desafios.2021.12.2.345

\section{RESUMEN}

Objetivo. Identificar los estilos de aprendizaje en estudiantes universitarios a partir de tres instrumentos: CHEA, KOLB Y VARK, desde la revisión descriptiva de la literatura. Métodos. Se realizó una búsqueda a través de bibliotecas virtuales: EBSCOhost, SciELO y ScienceDirect. Las palabras clave fueron: estilos de aprendizaje, universitarios, estudiantes, aprendizaje y sus combinaciones. Se recolectaron artículos cuyos instrumentos de medición fueron el cuestionario CHAEA, el inventario KOLB y el cuestionario VARK; resultando 20 artículos originales en idioma español. Resultados. En cuanto al uso del cuestionario CHAEA, los estudiantes de ciencias de la Salud presentaron un predominio por el estilo de aprendizaje reflexivo. En relación con el inventario KOLB, el estilo predominante en alumnos de medicina fue el convergente, así también, se apreció que el género femenino tiene una inclinación por el estilo divergente. En cuanto al uso del cuestionario VARK, la mayoría de los estudiantes de Ciencias sociales prefirieron un estilo de aprendizaje multimodal. Conclusiones. No se puede generalizar cuál es el estilo de aprendizaje por cada carrera o género, puesto que cada instrumento aborda diversos aspectos; además, las características socioculturales influyen en el tipo de aprendizaje de cada estudiante.

Palabras clave: estilos de aprendizaje; universitarios; aprendizaje; estudiantes.

\footnotetext{
ABSTRACT

Objective. To identify learning styles in university students from three instruments: CHEA, KOLB and VARK, from the descriptive review of literature. Methods. A search was made through virtual libraries: EBSCOhost, SciELO and ScienceDirect. The keywords were: learning styles, university students, learning and their combinations. Articles whose measuring instruments were the CHAEA questionnaire, the KOLB inventory and the VARK questionnaire were collected, resulting in 20 original articles in Spanish. Results. Regarding the use of the CHAEA questionnaire, the students of health sciences presented a predominance for the style of reflexive learning. In relation to the KOLB inventory, the predominant style in medical students was the convergent, so also, it was appreciated that the female gender has an inclination for the divergent style. Regarding the use of the VARK questionnaire, most social science students preferred a multimodal learning style. Conclusions. It is not possible to generalize which is the learning style for each career or gender, since each instrument addresses different aspects; in addition, the socio-cultural characteristics influence the type of learning of each student.

Keywords: learning styles; university students; learning; students.
} 


\section{INTRODUCCIÓN}

Los estilos de aprendizaje son aquellas características cognitivas, emocionales y físicas que expresan cómo los estudiantes interaccionan, interpretan y responden a su entorno de aprendizaje (Cardoso et al., 2021). Según Torales et al. (2018) son de tipo cognitivo ya que explican cómo aprende la persona; afectivo, debido a que está presente el deseo y la motivación de aprender. Así mismo, Bobadilla et al. (2017) definen a los estilos de aprendizaje como procesos subjetivos de percepción, integración, retención y aplicación de la información que las personas adquieren en base a sus experiencias del medio que los rodea.

Las personas tienen diferentes medios de percepción del ambiente; estos han sido motivo de estudio durante un largo tiempo. Las estrategias que se emplean para percibir los contenidos de aprendizaje se designan "estilos de aprendizaje". Saber cuál es el estilo de aprendizaje de los alumnos brinda dos ventajas principales; por una parte, se le permite al alumno capitalizar sus fortalezas (Juárez, 2013) y, por el otro, Viloria (2019) refiere que el profesor seleccionará diversas estrategias para la enseñanza y aprendizaje del estudiante a fin de elevar su educación.

Es evidente que la educación posee la capacidad de disminuir la pobreza, promover ámbitos más saludables e instaurar una cultura de innovación. Para que esto suceda la educación tiene que ser de calidad, con conocimientos relevantes, que sean pertinentes y con competencias que permitan a los alumnos afrontar los retos del mundo que los rodea; en tal sentido, en el "Marco de la Educación 2030", se proponen estrategias de aprendizaje a lo largo como es la inclusión y la equidad (Pezzini, 2016). Por ello, resulta importante conocer los estilos de aprendizaje en los estudiantes universitarios, conocer cómo comprenden y cómo aprenden, qué herramientas o estrategias aplican y sus resultados en el proceso de aprendizaje (Chambi et al., 2020). La educación es tan importante debido a que ayuda a la integración de los sujetos a su entorno social, posibilita el desarrollo de las habilidades cognitivas, afectivas y culturales (Almeida y Cunha, 2020).

Goguz y Ertek (2016) mencionan que el entorno que rodea al estudiante es fundamental, ya que se obtiene importantes beneficios en los resultados del aprendizaje. Por otro lado, Cascante y Villanueva (2020) declaran que los procesos de enseñanza y aprendizaje están vinculadas a una perspectiva holística de la interacción entre docentes y estudiantes. Por ello, la experiencia de ser docente universitario permite identificar las formas, mecanismos y estrategias con las que el docente capta la atención del estudiante. La vida universitaria es el espacio que utilizan para obtener o generar información a partir de experiencias vividas en las aulas; así también, para que tenga la capacidad de responder a actividades prácticas. Por lo tanto, se espera que los alumnos tengan un aprendizaje significativo y duradero que permita su progreso académico y, en el futuro, absolver dudas, incrementar sus saberes y solucionar problemas cotidianos como su práctica profesional (Chambi et al., 2020).

Cabe señalar que esta investigación permite, al profesional psicólogo educativo, a reconocer aquellos instrumentos que se utilizan para evaluar los estilos de aprendizaje y obtener la dimensión predominante de éste, propiciando el conocimiento de las conductas del estudiante asociadas al aprendizaje. Del mismo modo, dentro del ámbito educativo, busca apoyar a los profesionales en docencia a identificar aquellas habilidades que presenta el estudiante y así buscar técnicas y estrategias para la enseñanza y el aprendizaje, permitiendo afrontar con éxito la vida académica universitaria. El objetivo del estudio fue, por consiguiente, identificar los estilos de aprendizaje en estudiantes universitarios a partir de tres instrumentos: el cuestionario Honey-Alonso de estilos de aprendizaje (CHEA); el inventario de estilos de aprendizaje Kolb (KOLB) y el cuestionario visual, auditivo, lector-escritor y kinestésico de Fleming (VARK), desde la revisión descriptiva de la literatura.

\section{MÉTODOS}

Se realizó una exploración bibliográfica en tres bibliotecas virtuales, EBSCOhost, SciELO, ScienceDirect. Las palabras clave utilizadas fueron: estilos de aprendizaje, aprendizaje en universitarios y estudiantes y aprendizaje; así como sus combinaciones. Los criterios de inclusión fueron: artículos científicos originales, del año 2015 en adelante, en idioma español; a partir de lo cual, se recopilaron estudios latinoamericanos y de España, por el idioma. Fueron seleccionados aquellos artículos que utilizaron como instrumentos de medición el cuestionario CHAEA, el inventario KOLB y el cuestionario VARK, mientras que fueron excluidos los resúmenes de congresos, revisiones, estudios de caso y monografías o aquellos con otros instrumentos de medición. La justificación para el uso de estos tres instrumentos se basa en que estos son los más utilizados en la práctica psicoeducativa. Para el análisis de los datos se 
realizó un análisis cualitativo de tipo descriptivo y se elaboraron categorías de acuerdo con los instrumentos de recolección utilizados.

\section{RESULTADOS Y DISCUSIÓN}

A través de la revisión descriptiva de la literatura se encontraron 20 artículos científicos originales que se pueden apreciar a continuación (ver tabla 1).

\section{Tabla 1}

Resumen de los artículos encontrados en las bases de datos

\begin{tabular}{|c|c|c|c|}
\hline Autor & Tipo de estudio & Año & $\begin{array}{c}\text { Base de } \\
\text { datos }\end{array}$ \\
\hline Alducin y Vázquez & $\begin{array}{c}\text { Descriptivo- } \\
\text { transeccional- } \\
\text { no experimental }\end{array}$ & 2016 & ScIELO \\
\hline Almaguer et al. & Transversal & 2019 & EBSCOhost \\
\hline $\begin{array}{l}\text { Altamirano, Araya } \\
\text { y Paz }\end{array}$ & $\begin{array}{c}\text { Descriptivo- } \\
\text { transversal-relacional }\end{array}$ & 2019 & ScIELO \\
\hline Blanco & $\begin{array}{l}\text { Descriptivo- } \\
\text { correlacional }\end{array}$ & 2017 & SCIELO \\
\hline Bobadilla et al. & $\begin{array}{c}\text { Descriptivo- } \\
\text { transeccional- } \\
\text { no experimental }\end{array}$ & 2017 & SCIELO \\
\hline Caballero et al. & $\begin{array}{l}\text { Descriptivo- } \\
\text { correlacional- } \\
\text { transversal }\end{array}$ & 2020 & EBSCOhost \\
\hline $\begin{array}{l}\text { Carrasco y Gon- } \\
\text { zález }\end{array}$ & Transversal & 2018 & EBSCOhost \\
\hline Fernández & $\begin{array}{l}\text { Transversal- } \\
\text { observacional }\end{array}$ & 2019 & EBSCOhost \\
\hline $\begin{array}{l}\text { Freiberg, Ledesma } \\
\text { y Fernández }\end{array}$ & $\begin{array}{l}\text { Correlacional- } \\
\text { explicativo-transversal }\end{array}$ & 2017 & SciELO \\
\hline $\begin{array}{l}\text { Fuentes, Llermaly } \\
\text { y Silva }\end{array}$ & $\begin{array}{c}\text { No experimental- } \\
\text { descriptivo- } \\
\text { correlacional }\end{array}$ & 2018 & SCIELO \\
\hline $\begin{array}{l}\text { González, Sáez y } \\
\text { Ramirez }\end{array}$ & $\begin{array}{l}\text { Exploratorio- } \\
\text { comparativo }\end{array}$ & 2016 & SCIELO \\
\hline Jiménez et al. & Descriptivo- & 2020 & SCIELO \\
\hline Martín et al. & Descriptivo & 2020 & $\begin{array}{l}\text { ScienceDi- } \\
\text { rect }\end{array}$ \\
\hline $\begin{array}{l}\text { Marsiglia, Llamas y } \\
\text { Torregrosa }\end{array}$ & Descriptivo & 2020 & SCIELO \\
\hline $\begin{array}{l}\text { Martínez y } \\
\text { Delgado }\end{array}$ & $\begin{array}{l}\text { Transversal con dos co- } \\
\text { hortes independientes }\end{array}$ & 2017 & ScIELO \\
\hline Hernández & Analítica-interpretativa & 2016 & $\begin{array}{l}\text { Science } \\
\text { Direct }\end{array}$ \\
\hline Rodríguez et al. & Descriptiva & 2018 & $\begin{array}{l}\text { Science } \\
\text { direct }\end{array}$ \\
\hline Solari et al. & $\begin{array}{l}\text { Observacional- } \\
\text { transversal }\end{array}$ & 2019 & EBSCOhost \\
\hline $\begin{array}{l}\text { Tapia, Sánchez y } \\
\text { Vidal }\end{array}$ & $\begin{array}{l}\text { No experimental- } \\
\text { correlacional }\end{array}$ & 2020 & ScIELO \\
\hline Torales et al. & $\begin{array}{c}\text { Observacional-analítico } \\
\text { transversal- } \\
\text { no } \\
\text { probabilístico }\end{array}$ & 2018 & SciELO \\
\hline
\end{tabular}

Estilos deaprendizajeenestudiantesuniversitarioscon utilización del cuestionario de Honey-Alonso [CHAEA]

El cuestionario CHAEA evalúa 4 estilos de aprendizaje: activo, reflexivo, pragmático y teórico (González et al., 2016). Las personas de estilo activo están caracterizadas por ser creativas, espontáneas, impulsivas, entusiastas de personas competitivas que buscan resultados inmediatos y son de pensamiento abierta (Hernández et al., 2016). Las de estilo reflexivo son personas que analizan diferentes perspectivas para buscar una solución. En el estilo teórico son personas ordenadas y disciplinadas que buscan analizar, sintetizar y racionalizar todo; por último, las de estilo pragmático disfrutan de la realización práctica de ideas, gustan experimentarlas, toman decisiones rápidas, actúan de manera inmediata y con seguridad (Caballero et al., 2020; Martínez y Delgado, 2017; Jiménez et al., 2019).

El cuestionario cuenta con 20 ítems por cada estilo de aprendizaje, haciendo un total de 80 preguntas. Las respuestas son dicotómicas: el signo positivo (+), de acuerdo; y el negativo (-), desacuerdo. Los materiales para su aplicación son: manual, hoja de aplicación y hoja de perfil de aprendizaje (Adulcin y Vázquez, 2016). La aplicación es de forma individual y grupal. El estilo predominante se obtiene al sumar el signo positivo de cada estilo, siendo 20 el puntaje máximo. Los grados de inclinación son: muy baja, baja, moderada, alta y muy alta. El instrumento tiene una confiabilidad de un $\alpha$ 0,80 (Caballero et al., 2020; Torales et al., 2018). A continuación, los artículos recopilados (ver tabla 2).

A partir de los artículos, se observó que Altamirano et al. (2019) y Carrasco y Gonzáles (2018) estudiaron las variables estilos de aprendizaje y rendimiento académico. Los resultados evidenciaron que existe una correlación entre ambas variables; así también, los estudiantes que poseen el estilo reflexivo presentan un rendimiento académico superior. El estudio de Carrasco y Gonzáles (2018) demostró una relación inversa entre estilo activo y el rendimiento académico, siendo que a mayor rendimiento académico menor estilo activo de aprendizaje.

En cuanto a los estilos de aprendizaje en alumnos de Psicología, se obtuvieron diferentes resultados; siendo que, en el estudio de Bobadilla et al. (2017) se mostró una preferencia moderada por el estilo activo, en tanto que Carrasco y Gonzáles (2018) evidenciaron que los estudiantes de Psicología presentan los cuatro estilos de aprendizaje con una preferencia moderada; asimismo, en el estudio de Freiberg et al. (2017) se encontró que los estudiantes de Psicología obtuvieron una baja preferencia por el estilo 
Tabla 2

Artículos científicos que utilizaron el cuestionario Honey-Alonso (CHAEA)

\begin{tabular}{|c|c|c|c|c|}
\hline Título del artículo & $\begin{array}{l}\text { Tamaño de } \\
\text { muestra }\end{array}$ & Hombres & Mujeres & Edades \\
\hline $\begin{array}{l}\text { Autoevaluación de conocimientos previos y rendimiento según } \\
\text { estilos de aprendizaje en un grado universitario de edificación }\end{array}$ & 161 & 102 & 59 & $18-30$ \\
\hline $\begin{array}{l}\text { Estilos y estrategia de enseñanza-aprendizaje de estudiantes } \\
\text { universitarios de la Ciencia del Suelo }\end{array}$ & 65 & 39 & 26 & 21 promedio \\
\hline $\begin{array}{l}\text { Estilos de aprendizaje, elección de carrera y perfil curricular en } \\
\text { estudiantes de Comunicación Humana }\end{array}$ & 166 & 22 & 148 & $17-31$ \\
\hline $\begin{array}{l}\text { Estilos de aprendizaje y rendimiento académico de estudiantes } \\
\text { de la carrera de Obstetricia }\end{array}$ & 208 & 8 & 200 & $18-20$ \\
\hline $\begin{array}{l}\text { Estilos de aprendizaje en estudiantes de la licenciatura en Psico- } \\
\text { logía del centro universitario UAEM Temascaltepec, } 2016\end{array}$ & 311 & 68 & 243 & $17-23$ \\
\hline $\begin{array}{l}\text { Estilos de aprendizaje y rendimiento académico en estudiantes } \\
\text { de Enfermería durante evaluaciones de simulación clínica }\end{array}$ & 68 & 13 & 55 & $18-32$ \\
\hline $\begin{array}{l}\text { Influencia del estilo de aprendizaje y del tipo de tarea en los } \\
\text { procesos de búsqueda en línea de estudiantes universitarios }\end{array}$ & 92 & 11 & 81 & $18-19$ \\
\hline $\begin{array}{l}\text { Estilos de aprendizaje de estudiantes de Medicina de primer año } \\
\text { de la Universidad Nacional de Asunción, Paraguay }\end{array}$ & 181 & 83 & 98 & $17-14$ \\
\hline $\begin{array}{l}\text { Estilos de aprendizaje en Fonoaudiología y Terapia Ocupacional } \\
\text { en la Universidad de Antofagasta, Chile }\end{array}$ & 141 & - & - & - \\
\hline $\begin{array}{l}\text { Estilos de aprendizaje y rendimiento académico en estudiantes } \\
\text { universitarios de Psicología en Talca }\end{array}$ & 164 & 113 & 51 & - \\
\hline $\begin{array}{l}\text { Estilos de aprendizaje en estudiantes de Medicina de la Universi- } \\
\text { dad Walter Sisulu de Sudáfrica }\end{array}$ & 72 & - & - & $17-25$ \\
\hline $\begin{array}{l}\text { Estilos y estrategias de aprendizaje en estudiantes universitarios } \\
\text { de Buenos Aires }\end{array}$ & 438 & 262 & 176 & $19-36$ \\
\hline $\begin{array}{l}\text { Perfiles de estilos de aprendizaje y rendimiento académico en } \\
\text { estudiantes de primer año de Enfermería }\end{array}$ & 193 & - & - & - \\
\hline
\end{tabular}

pragmático, cuando se trata de emplear estrategias de motivación de aprendizaje.

Por otro lado, se demostró que los estudiantes de Medicina presentaron un estilo de aprendizaje reflexivo según Torales et al. (2018). Así también, Altamirano et al. (2019) evidenciaron que los alumnos de Obstetricia presentan el estilo reflexivo con una mayor predominancia; mientras que Fernández (2019) evidenció que los estilos reflexivo, teórico y pragmático son los más desarrollados por los estudiantes de Ciencias de la Salud, Io cual concuerda con un trabajo realizado en Colombia por Bueno et al. (2017), quien halló una relación estadísticamente significativa entre las competencias de atención en salud y el estilo de aprendizaje reflexivo. Para instituciones de formación profesional en carreras de salud, saber aplicar el estilo de aprendizaje reflexivo cuando de competencias de atención se trata, abre un campo de actividades para que los alumnos puedan llegar a desenvolverse con mayor destreza.

En cuanto al estilo de aprendizaje y Género; Torales et al. (2018), evidenciaron que existe relación entre los estilos reflexivo, pragmático y el género masculino. Asimismo, Freiberg et al. (2017) encontraron que el género masculino tuvo una mayor tendencia por el estilo pragmático. Por otra parte, cabe mencionar que en ambas investigaciones no existió una relación significativa entre los estilos de aprendizaje y el género femenino.

En un estudio realizado en México, se corroboró que el estilo de aprendizaje fue el teórico, seguido del reflexivo, pragmático y, finalmente, el activo; lo cual muestra que los estudiantes difieren en su elección de estilo de aprendizaje (Ortega, 2013). Se considera que cada estudiante tiene un estilo predominante de aprendizaje e, inclusive, puede aplicar diferentes estilos según la asignatura; aun así, si estos estilos no son utilizados ni desarrollados de manera adecuada no repercute en el rendimiento académico (Palomino, 2020). Para los docentes, no solo es importante considerar los aspectos temáticos de una asignatura, sino también determinar cuál es la mejor estrategia para abordar los contenidos, así también seleccionar y utilizar procedimientos para un mejor aprendizaje como la retroalimentación de la información en el proceso enseñanza-aprendizaje (Solari et al.,2019). 
Por ello es importante abordar estos dos puntos de forma articulada, aunque no solo basta el estilo de aprendizaje, sino también el contexto socioeducativo-cultural donde se desarrollen (Marsiglia et al., 2020).

\section{Estilos deaprendizajeenestudiantesuniversitarioscon utilización del inventario KOLB}

El inventario de estilos de aprendizaje KOLB está formado por 48 ítems y evalúa cuatro estilos: convergente, divergente, asimilador y acomodador. El estilo convergente hace referencia a personas que toman buenas decisiones y prefieren solucionar los problemas de forma práctica. En el estilo divergente están las personas que les gusta observar desde distintas perspectivas y son buenos brindando ideas. En el estilo asimilador se encuentra aquellas personas que prefieren la soledad y se centran en ideas y conceptos abstractos. Por último, el estilo acomodador corresponde a aquellas personas flexibles e intuitivas que aprenden de sus experiencias y buscan tomar riesgos. (Caballero et al., 2020; Tapia et al.,2020).

Las puntuaciones oscilan del 1 al 4; se coloca 4 puntos a la situación que se informe más beneficiosa para el evaluado mientras que 3 , 2 y 1 se coloca para las restantes situaciones menos beneficiosas. Para determinar el estilo de aprendizaje se procede con el conteo de la hoja de respuestas en forma vertical, dando como resultado un número determinado. Luego se coloca en las cuadrantes EC: Experimentación concreta, OR: observación reflexiva, CA: conceptuación abstracta y EA: experimentación activa. Cuanto más cerca esté el punto de intersección de los ejes CA-EC y EA-OR, más equilibrado será su estilo de aprendizaje. El cuestionario tiene una fiabilidad que fluctúa de acuerdo a un alpha de Cronbach entre 0,67 y 0,87. (Caballero et al., 2020; Rodríguez et al., 2018, Morales y Rojas, 2016).

A continuación, se revisan los artículos analizados donde utilizaron el estilo de aprendizaje KOLB (ver tabla 3). En la investigación realizada por Tapia et al. (2020) se encontró que los estilos de aprendizajes predominantes fueron el convergente y el asimilador. Para este estudio fueron evaluados universitarios de diversas carreras que hacian del sitio web YouTube una herramienta de aprendizaje y motivación. Así mismo, Rodríguez et al. (2018) mencionan que el estilo predominante fue el convergente en el estudio realizado con alumnos de Medicina Humana. En cambio, en los resultados de Caballero et al. (2020) mencionaron que los
Tabla 3

Estilos de aprendizaje en estudiantes universitarios con utilización del cuestionario KOLB

\begin{tabular}{|c|c|c|c|c|}
\hline Título del artículo & $\begin{array}{c}\text { Tamaño de } \\
\text { muestra }\end{array}$ & Hombres & Mujeres & Edades \\
\hline $\begin{array}{l}\text { Tipos psicológicos y } \\
\text { estilos de aprendizaje } \\
\text { en estudiantes de la } \\
\text { carrera de Odontología } \\
\text { en la Universidad de la } \\
\text { Frontera, Chile }\end{array}$ & 372 & 164 & 208 & - \\
\hline $\begin{array}{l}\text { Análisis de los estilos de } \\
\text { aprendizaje en alumnos } \\
\text { de Medicina de la } \\
\text { Universidad de Chile }\end{array}$ & 101 & 63 & 38 & 18 \\
\hline $\begin{array}{l}\text { Estilos de aprendizaje } \\
\text { e intención de uso de } \\
\text { videos académicos de } \\
\text { YouTube en el contexto } \\
\text { universitario chileno }\end{array}$ & 235 & 108 & 127 & $18-22$ \\
\hline $\begin{array}{l}\text { Estilos de aprendizaje y } \\
\text { rendimiento académico } \\
\text { en estudiantes de } \\
\text { Enfermería durante } \\
\text { evaluaciones de } \\
\text { simulación clínica }\end{array}$ & 68 & 13 & 55 & $18-32$ \\
\hline $\begin{array}{l}\text { Estilos de aprendizaje } \\
\text { y actitudes ante la } \\
\text { investigación científica } \\
\text { en estudiantes univer- } \\
\text { sitarios }\end{array}$ & 195 & - & - & $23-30$ \\
\hline
\end{tabular}

estudiantes presentan mayor predominancia al estilo de aprendizaje acomodador con un 42,65\%. Sin embargo, Blanco (2017) y los estudios de Fuentes et al. (2018), encontraron que el estilo de aprendizaje preeminente en los estudiantes es el divergente.

Una persona asimiladora es caracterizada por la observación reflexiva, es decir, por juntar observaciones dispares en una explicación integral; en tanto que el estilo convergente se caracteriza por la aplicación práctica de las ideas, por dar una solución concreta a un problema o pregunta (Matzumura et al., 2018).

En el estudio de Fuentes et al. (2018) se observó que el género femenino tiene una mayor inclinación al estilo de aprendizaje divergente. Sin embargo, en la investigación realizada por Tapia et al. (2020) se encontró que los estilos predominantes en ambos géneros son el convergente y el asimilador; asimismo, se encontró que, en las áreas de economía y administración, humanidades e ingeniería, los estudiantes tienen preferencia por el estilo convergente

En un estudio realizado en Perú con alumnos de Medicina Humana se encontró que la mayoría tenía un estilo de aprendizaje asimilador, como mencionan los autores Matzumura et al. (2018). 
El modelo de estilos de aprendizaje KOLB se fundamenta en la teoría integral sobre el aprendizaje y el desarrollo, donde la vital fuente de aprendizaje lo constituye la experiencia; al ser la carrera de Medicina una profesión donde se trabajan con diferentes informaciones (paciente, análisis, antecedentes), el alumno tiene que integrar las informaciones y dar una respuesta integral (Martín et al., 2020). Por otro lado, se encontró un estudio realizado por Villalba (2018), sobre experiencias discursivas y Lingüisticas, donde se encontró que el estilo de aprendizaje divergente se relaciona con niveles sobresalientes de estructura textual; así también, mostraron ser más creativos. A raíz de los dos estudios se puede considerar que el estilo de aprendizaje depende de los conocimientos que se pretenden impartir para utilizar una dinámica diferente de enseñanza basada en la carrera y el propósito final.

\section{Estilos de aprendizaje en estudiantes universitarios con utilización del Cuestionario VARK de Fleming}

De todos los artículos analizados, solo dos de ellos trabajan con el cuestionario VARK de Fleming (ver tabla 4). El cuestionario VARK identifica las preferencias de los estudiantes mediante cuatro estilos de aprendizaje sensoriales: visual (V), auditivo (A), lectura/escritura (R) y kinestésico (K). Consta de 16 items con cuatro tipos de respuestas. Se reportaron niveles satisfactorios de validez y consistencia para el VARK. (Almaguer et al., 2019; Marsiglia et al., 2020).

En la investigación realizada por Almaguer et al. (2019) se demostró que la mayoría de los estudiantes de Ciencias Sociales, prefieren un estilo multimodal (visual, auditivo, lectura/escritura y kinestésico). A diferencia de Marsiglia et al. (2020) quienes obtuvieron como resultado que los estilos, lector con $31,7 \%$ y auditivo con un 28,6 \% tienen mayores porcentajes de preferencia entre los estudiantes de licenciatura en Educación.

La correlación entre género y estilos de aprendizaje muestra, según Marsiglia et al. (2020), el género masculino y femenino tienen preferencias por el estilo de aprendizaje lector; sin embargo, en el estudio de Almaguer et al. (2019) se halló que no existe asociación significativa entre el género y los estilos de aprendizaje, más bien se aprecia un estilo bimodal auditivo y lector.

En ese contexto, Espinoza et al. (2019) realizaron un estudio con estudiantes universitarios con el propósito examinar los estilos de aprendizaje de los estudiantes de pregrado de una universidad privada de la ciudad de Chiclayo, mostrando que el estilo de aprendizaje es variable con la edad, resultando que entre los estudiantes más jóvenes, entre 18 y 23 años, predomina un solo estilo de aprendizaje, por el contrario, los estudiantes adultos poseen varios estilos de estudio; además, se llegó a la conclusión de que el estilo de aprendizaje es multimodal y cada uno de los estilos y su aplicación brinda un aporte significativo en la educación.

En conclusión, se encontró que la mayoría de los estudios aplicaron el instrumento CHAEA de Honey-Alonso, siendo la dimensión reflexiva como el resultado predominante en estudiantes de Ciencias de la Salud. Además, se pudo apreciar que el instrumento presenta carencias de homogeneidad de sus elementos; por ello sería necesario realizar una revisión de sus propiedades psicométricas, ya que este instrumento es utilizado por varios autores con el fin de evaluar los estilos de aprendizaje.

Asimismo, se encontró que los alumnos de Ciencias de la Salud tienen mayor afinidad por el tipo de aprendizaje reflexivo, los estudiantes de Ciencias Administrativas e Ingenierías tienen un estilo convergente mientras que en Humanidades y Arquitectura tienen un estilo asimilador. Así también, los alumnos de Ciencias Sociales y Pedagogía tienen una preferencia por el estilo auditivo.

Los resultados obtenidos en cuanto al estilo de aprendizaje no se pueden generalizar puesto que un estilo de aprendizaje también está mediado por otras variables como rasgos de personalidad, rendimiento académico y la realidad social

\section{Tabla 4}

Estilos de aprendizaje en estudiantes universitarios con utilización del cuestionario VARK

\begin{tabular}{lcc}
\hline Título del artículo & Tamaño de muestra & Hombres \\
\hline $\begin{array}{l}\text { Estilos de aprendizaje de preferencia entre estudiantes de } \\
\text { Medicina en La Gambia }\end{array}$ & 113 & 54 \\
$\begin{array}{l}\text { Las estrategias de enseñanza y los estilos de aprendizaje una } \\
\text { aproximación al caso de la licenciatura en educación de la } \\
\text { Universidad de Cartagena (Colombia) }\end{array}$ & 30 & 10 \\
\hline
\end{tabular}


y cultural de los participantes. El proceso de enseñanza-aprendizaje lo asume el estudiante y el docente. Cada estudiante tiene un estilo predominante de aprendizaje e, inclusive, puede aplicar diferentes estilos según la asignatura; sin embargo, al no ser utilizados debidamente o no desarrollarlos, estos estilos repercuten en el rendimiento académico.

El trabajo llega a la conclusión de que no se puede generalizar cuál es el estilo de aprendizaje preciso por cada carrera. Así también, no se puede elegir un instrumento de recolección como patrón de referencia, puesto que cada instrumento aborda diversos aspectos dentro de los estilos de aprendizaje y estos son mediados por diversos factores socioculturales que determinarán su estilo y su en el proceso de enseñanza-aprendizaje.

Se recomienda realizar estudios en jóvenes universitarios del Perú, para hacer una exploración de estos y analizar, de acuerdo a la realidad peruana, cuales son los estilos de aprendizaje que más se adecuan a nuestro nivel socioeducativo y cultural.

\section{REFERENCIAS}

Alducin, J. y Vázquez, M. (2016). Autoevaluación de conocimientos previos y rendimiento según estilos de aprendizaje en un grado universitario de edificación. Formación Universitaria, 9(2), 29-40. https:// doi.org/10.4067/S0718-50062016000200004

Almaguer, L., Avila, M., Sánchez, Y., Senghore, T., Sarr, F. y Nyan, O. (2019). Estilos de aprendizaje de preferencia entre estudiantes de Medicina en La Gambia. Educación Médica Superior, 33(4), 37-55. http://www.ems.sld.cu/index.php/ems/article/ view/1731/906

Almeida, N. y Cunha, A. (2020). Un debate integrador sobre los estilos de aprendizaje y el proceso de aprendizaje. Social Sciences \& Humanities Open, 1(2). https://doi.org/10.1016/j.ssaho.2020.100017

Altamirano, J., Araya, S. y Paz, M. (2019). Estilos de aprendizaje y rendimiento académico de estudiantes de la carrera de obstetricia. Revista Ciencias de la Salud, 17(2), 276-292. https://doi.org/10.12804/revistas. urosario.edu.co/revsalud/a.7937

Blanco, A. (2017). Estilos de aprendizaje y actitudes ante la investigación científica en estudiantes universitarios. Artículo de reflexión Investigación y desarrollo, 25(2), 82-99. https://dx.doi.org/10.14482/indes.25.2.10960

Bueno, Y. T., Contreras, A. R., Gómez, A. y Jacome, Z. Z. (2017). Asociación entre estilos de aprendizaje, rendimiento académico y resultados de las pruebas SABER PRO en los estudiantes del programa de fisioterapia Universidad de Santander del último año, Bucaramanga, 2017 [Tesis de pregrado, Universidad de San- tander]. Repositorio de Universidad de Santander. https://repositorio.udes.edu.co/handle/001/100

Bobadilla, S., Cardoso, D., Carreño, L. y Márquez, J. (2017). Estilos de aprendizaje en estudiantes de la licenciatura en psicología del centro universitario UAEM Temascaltepec, 2016. RIDE Revista Iberoamericana para la Investigación y el Desarrollo Educativo, 7(14), 34-50. https://doi.org/10.23913/ride.v7i14.271

Caballero, E., Avendaño, M., Busquets, P., Hernández, A. y Astorga, C. (2020). Estilos de aprendizaje y rendimiento académico en estudiantes de enfermería durante evaluaciones de simulación clínica. Revista Cubana de Enfermería, 36(4), 1-14. http:// search.ebscohost.com/login.aspx?direct=true\&d$b=a 9 h \& A N=148445088 \&$ lang=es\&site=ehost-live

Cardozo, L., Rodrigues, D., Molano, E., Moreno, J., Beltran, C. y Borrero, F. (2021) Propiedades psicométricas del cuestionario de estilos de aprendizaje "CHAEA-36" en estudiantes universitarios. Estudios sobre educación, 40, 75-101. https://doi. org/10.15581/004.40.75-101

Carrasco, F. y González, M. (2018). Estilos de aprendizaje y rendimiento académico en estudiantes universitarios de Psicología en Talca. Revista UCMaule, 54, 107-132. https://doi.org/10.29035/ucmaule.54.107

Chambi, A., Cienfuegos, J. y Espinoza., T. (2020). Estilos de aprendizaje y rendimiento académico en internos de enfermería de una universidad pública peruana. Revista de La Facultad de Medicina Humana, 20(1), 43-50. https://doi.org/10.25176/rfmh.v20i1.2546

Cascante, N., y Villanueva, L. (2020). Formación docente en didáctica universitaria en la pandemia: entre la reflexión pedagógica y la instrumentalización. Costa Rica .Investigaciones y Experiencias, 7(2), 109-117. http://www.scielo.edu.uy/pdf/ic/v7n2/2301-0126ic-7-02-107.pdf

Espinoza, J., Miranda, W. y Chafloque, R., (2019). Los estilos de aprendizaje Vark en estudiantes universitarios de las escuelas de negocios. Revista de Psicología Educativa, 7(2), 384-414. http://dx.doi.org/10.20511/ pyr2019.v7n2.254

Fernández, R. (2019). Estilos de aprendizaje en estudiantes de Medicina de la Universidad Walter Sisulu de Sudáfrica. Revista Cubana de Educación Médica Superior, 33(2), 1-12. http://search.ebscohost.com/login. aspx? direct = true $\& \mathrm{db}=$ a 9h\&AN = 141714779\&lan$\mathrm{g}=$ es\&site = ehost-live

Freiberg, A., Ledesma, R. y Fernández, M. (2017). Estilos y estrategias de aprendizaje en estudiantes universitarios de Buenos Aires. Revista de Psicología (Perú), 35(2), 535-573. https://doi.org/10.18800/ psico.201702.006

Fuentes, J., Llermaly, S. y Silva, M. (2018). Tipos estilos de aprendizaje en psicológicos y estudiantes de la carrera de Odontología en la Universidad de La Frontera, Chile. En Int. J. Odontostomat, 12(2), 182-187. http://dx.doi. org/10.4067/S0718-381X2018000200182

Gogus, A. y Ertek, G. (2016). Atributos personales y de aprendizaje de los estudiantes universitarios en la predicción y clasificación de los estilos de aprendizaje: estilos de aprendizaje de nueve regiones y cuatro 
regiones de Kolb. Procedia Ciencias Sociales y del Comportamiento, 217, 779-789. https://doi.org/10.1016/j.sbspro.2016.02.145

González, F., Sáez, K. y Ramírez, J. (2016). Perfiles de estilos de aprendizaje y rendimiento en primer año de enfermería. Ciencia y Enfermería, 22(1), 87-99. http:// dx.doi.org/10.4067/S0717-95532016000100008

Hernández, M., Serate, S. y Campos, R. (2016). Influencia del estilo de aprendizaje y del tipo de tarea en los procesos de búsqueda en línea de estudiantes universitarios. Investigación Bibliotecológica: archivonomía, bibliotecología e información, 29(65), 115-136. doi: http://dx.doi.org/10.1016/j.ibbai.2016.02.017

Jiménez, L., Vega, N., Capa, E., Fierro, N. y Quichimbo, P. (2019). Estilos y estrategia de enseñanza-aprendizaje de estudiantes universitarios de la Ciencia del Suelo Learning. Revista Electrónica de Investigación Educativa, 21(1), 1-10. https://doi.org/10.24320/redie.2019.21.e04.1935

Juárez, S., (2013). Estilos de aprendizaje en estudiantes de pregrado y posgrado del Hospital General Regional del Instituto Mexicano del Seguro Social, Investigación en Educación Médica, 2(1), 12-24. https://doi. org/10.1016/S2007-5057(13)72678-4

Marsiglia, R., Llamas C. y Torregroza, E. (2020). Estrategias de enseñanza y estilos de aprendizaje una aproximación al caso de la licenciatura en educación de la Universidad de Cartagena (Colombia). Formación universitaria, 13(1), 27-34. https://dx.doi. org/10.4067/S0718-50062020000100027

Martínez, G. y Delgado, U. (2017). Estilos de aprendizaje, elección de carrera y perfil curricular en estudiantes de Comunicación Humana. Revista Digital de Investigación en Docencia Universitaria, 11(2), 274-287. https://doi.org/10.19083/ridu.11.526

Martín, J., Toledo, E., Martínez, P., Ruíz, J., Fernández, R., López, A., Manuel, J. y Rodríguez, J. (2020). Análisis de los estilos de aprendizaje en un curso de habilidades técnicas laparoscópicas. Implicaciones para el entrenamiento quirúrgico, 23. https://doi. org/10.1016/j.ciresp.2020.11.006

Matzumura, J., Gutiérrez, H., Pastor, C., Zamudio, L. y Ruiz, R., (2018). Metodología activa y estilos de aprendizaje en el proceso de enseñanza en el curso de metodología de la investigación de una facultad de ciencias de la salud. Anales de la facultad de Medicina, 79(4), 293-300. http://dx.doi.org/10.15381/ anales.v79i4.15632

Solari, G., Rivera, M., Álvarez, M. y Velasco, A., (2019). Estilos de aprendizaje en Fonoaudiología y Terapia Ocupacional en la Universidad de Antofagasta, Chile. Revista Cubana de Educación Médica Superior, 33(2), 1-16. http:// web.b.ebscohost.com/ehost/pdfviewer/pdfviewer?$\mathrm{vid}=10 \mathrm{gsid}=646 \mathrm{dfd} 78-9 \mathrm{f64}-4297-801 \mathrm{e}-306 \mathrm{cf}-$ 736519d\%40pdc-v-sessmgr02

Morales, Y. y Rojas, J. (2016). La personalidad y el estilo de aprendizaje: una relación mutua en el rendimiento académico en la clase de inglés. Lenguas Modernas, 24, 9-12. DOI 10.15517/RLM.VOI24.24688

Ortega, E. (2013). Modificación y aplicación del Cuestionario Honey Alonso sobre Estilos de Aprendizaje
(CHAEA), en una muestra de alumnos de la carrera de QFB de la FES Zaragoza [Tesis de pregrado, Universidad Nacional Autónoma De México]. Repositorio Institucional de la UNAM. https://condor. zaragoza.unam.mx/portal/wp-content/Portal2015/ Licenciaturas/qfb/tesis/tesis_ortega_lopez.pdf

Palomino, K. (2020). Estilo de aprendizaje reflexivo y burnout académico en maestrandos de Docencia e Investigación en Salud. Desafios,11(2), 121-8. https://doi. org/10.37711/desafios.2020.11.2.207

Pezzini, M. (2016). Avanzado hacia una mejor educación para Perú [Archivo PDF]. Organización para La Cooperación y Desarrollo Económicos (OCDE). https:// www.oecd.org/dev/Avanzando-hacia-una-mejor-educacion-en-Peru.pdf

Rodríguez, H., Pirul, J., Robles, J., Pérez, L., Vásquez, E., Galaz, I., Cuellar, C., Díaz, H. y Arriaza, C. (2018). Análisis de los estilos de aprendizaje en alumnos de Medicina de la Universidad de Chile. Educación Médica, 19(1), 2-8. https://doi.org/10.1016/J.EDUMED.2016.11.004

Tapia, J., Sánchez, A., y Vidal, C. (2020). Estilos de aprendizaje e intención de uso de videos académicos de YouTube en el contexto universitario chileno. Formación Universitaria, 13(1), 3-12. https://doi.org/10.4067/ s0718-50062020000100003

Torales, J., Barrios, I., Villalba, J., Camacho, C., Gomez, J., San Miguel, C., Giménez, E. y Aparicio, C. (2018). Estilos de aprendizaje de estudiantes de Medicina de primer año de la Universidad Nacional de Asunción, Paraguay. An. Fac. Cienc. Méd. (Asunción), 51(02), 27-36. http://scielo.iics.una. py//scielo.php?script=sci_arttext\&pid=\$1816-89492018000200027\&lang=en

Viloria, A., Petro, I. y Pineda, O., (2019). Preferencia de estilos de Aprendizaje de los estudiantes universitarios que utilizan Big Data. Procedia Ciencias de la Computación, 160, 461-466. https://doi.org/10.1016/j. procs.2019.11.064

Villalba, N. (2018). Los estilos de aprendizaje y la competencia argumentativa escrita bilingüe (español-inglés) en términos de las escogencias discursivas y lingüísticas. Lenguaje, 46(2), 266-291. https://doi.org/10.25100/ lenguaje.v46i2.6583

\section{Contribución de los autores}

CTPM: concepción, redacción del contenido y análisis del contenido.

IACA: concepción, redacción del contenido y análisis del contenido.

MQP: concepción, redacción del contenido y análisis del contenido.

CRC: diseño, revisión metodológica y guía del trabajo en general.

\section{Fuentes de financiamiento}

La investigación fue realizada con recursos propios.

\section{Conflictos de interés}

Los autores declaran no tener conflicto de interés.

\section{Correspondencia}

Cira Thania Porras Mucha

Huancayo/Huancayo,

CP.:12731

Cel.: 916628980

ciraporras1@gmail.com 